\title{
Changes in the fatty acid composition of Phaeodactylum tricornutum and Dunaliella tertiolecta during growth and under phosphorus deficiency
}

\author{
Robert Siron $^{1, *}$, Gérard Giusti $^{1}$, Brigitte Berland ${ }^{2}$ \\ ${ }^{1}$ Centre d'Océanologie de Marseille, Faculté des Sciences de Luminy, F-13288 Marseille cédex 09, France \\ ${ }^{2}$ Station Marine d'Endoume, Rue de la Batterie des Lions, F-13007 Marseille, France
}

\begin{abstract}
Total fatty acid content increased with age in batch cultures of Phaeodactylum tricornutum. Chromatographic analysis revealed changes in the fatty acid composition of the diatom grown in natural enriched medium. Particularly noticeable was the storage of palmitic and palmitoleic acids by the end of the growth period, to the detriment of eicosapentaenoic acid. This study also gives evidence of the importance of oleic acid as a precursor in the biosynthesis of long-chain polyunsaturated fatty acids in this diatom. Phosphorus limitation also led to a significant increase in the total fatty acid cell content of $P$. tricornutum and Dunaliella tertiolecta. When $P$. tricornutum was cultured in phosphorusdeficient medium, the fatty acid composition was similar to that of a senescent batch culture. When division rate was reduced, oleic acid content of $D$. tertiolecta increased, confirming this fatty acid as an intermediate component in the lipid metabolism of this chlorophyte during the division processes
\end{abstract}

\section{INTRODUCTION}

Although fatty acids represent a small proportion of the total organic matter synthesized by marine microalgae, they nevertheless remain an important source of energy for marine trophic chains. Their importance is also qualitative since some phytoplanktonic fatty acids are essential to higher trophic levels (Ackman 1967, Kanazawa et al. 1979). Phytoplanktonic fatty acids were thus investigated early in taxonomic (Ackman et al. 1968, Wood 1974), energetic (Lee et al. 1971, Siron \& Giusti 1985) and biogeochemical studies (Schneider et al. 1970, Saliot \& Barbier 1973, Mayzaud et al. 1976). More recently, the fatty acid composition of microalgae was shown to be altered by environmental changes and especially by different nutrient supplies. Since nitrogen is generally the limiting factor of algal productivity in the open ocean, most studies focus on nitrogen limitation (Pohl \& Zurheide 1979, Tornabene et al. 1983, Piorreck et al. 1984, Suen et al. 1987). A few studies are

\footnotetext{
- Present address: Laboratoire d'Océanologie de Rimouski (INRS), 310, des Ursulines, Rimouski Québec, Canada G5L $3 \mathrm{~A} 1$
}

also concerned with silica deficiency (Shifrin \& Chisholm 1981, Taguchi et al. 1987) or variable total nutrient supply (Mayzaud unpubl.), but we are not aware of any study concerning phosphorus limitation. Yet, phosphorus can be the main limiting factor to algal production in some coastal areas (Berland et al. 1980, Myers \& Iverson 1981, Kaellqvist 1988). The aim of this work is to supplement available data concerning the influence of environmental changes on the biosynthesis of fatty acids, with emphasis on phosphorus deficiency. Although these preliminary results obtained in batch cultures cannot be directly extrapolated to natural conditions, they contribute to ascertaining general patterns in fatty acid biosynthesis of marine microalgae related to nutrient limitations.

\section{MATERIALS AND METHODS}

Organisms. Phaeodactylum tricornutum Bohlin (diatom) and Dunaliella tertiolecta Butcher (chlorophyte) were obtained from the collection of the Station Marine d'Endoume, France.

Growth conditions. Experiments were conducted in 
1 l cotton-stoppered flasks under continuous light exposure $\left(35 \mu \mathrm{E} \mathrm{m}^{-2} \mathrm{~s}^{-1}\right)$ provided by banks of natural white fluorescent tubes (Sylvania Grolux) and at a temperature of $18 \pm 1{ }^{\circ} \mathrm{C}$. All flasks of the same experiment were inoculated from an axenic preculture in exponential growth phase, acclimated to the same ambient conditions. Each inoculum was adjusted to an initial concentration of about $5 \times 10^{3}$ cell ml $\mathrm{m}^{-1}$, consistent with cell densities generally found in eutrophic coastal areas and allowing the investigation of all growth phases in enriched batch cultures (Siron et al. 1986).

Growth medium. Algae were cultured in natural enriched medium. Seawater was collected offshore in the Mediterranean Sea and subsequently filtered through $0.22 \mu \mathrm{m}$ membranes before use. Salinity was reduced to $33 \%$ and $\mathrm{pH}$ adjusted to 7.4 with dilute $\mathrm{HCl}$ solution to provide optimal growth conditions in batch cultures (Berland 1966). Nutrient supply used in this work was previously detailed by Antia et al. (1975). The phosphorus-sufficient medium (PS) was supplemented with $\mathrm{NaH}_{2} \mathrm{PO}_{4}\left(6.9 \mathrm{mg} \mathrm{l^{-1 }}\right)$ while in phosphorus-deficient medium (PD) no phosphate was added; negligible quantities ( $<0.2 \mathrm{mg} \mathrm{PO}_{4} \mathrm{l}^{-1}$ ) were introduced from the inoculation. Experiments were conducted in axenic conditions by autoclaving test media before inoculation.

Growth measurements. Microscopic cell counts were done on aliquots of culture suspensions using Nageotte and Neubauer haemocytometer chambers. In order to minimize counting errors, a minimum of 300 cells per sample were counted. Reported values are means of triplicate counts. Division rate $(k)$ was calculated by the following formula:

$$
k=\ln \left(N_{1} / N_{0}\right) \times\left(1.443 /\left[t_{1}-t_{0}\right]\right)
$$

where $N_{0}$ and $N_{1}=$ cell densities at times to and $t_{1}$ respectively.

Lipid analysis. Cells were harvested by filtration through pre-extracted and tared fiberglass filters (Whatman GF/C). Filters were then washed with an ammonium formate solution isotonic to seawater to remove excess sea salts, dried overnight at $40^{\circ} \mathrm{C}$ and weighed. Cells were extracted in a Soxhlet apparatus using a chloroform:methanol (2:1) mixture, refluxing for $24 \mathrm{~h}$. Solvents were then evaporated under reduced pressure and the residue was dried under slight nitrogen current. The total lipid extract was saponified by a sodium methoxide (MeONa) solution for $15 \mathrm{~min}$ according to the method of Luddy et al. (1960). Unsaponifiable matter (USM) was dissolved in a small volume of $n$-hexane and removed from other lipids. After evaporation of solvent, the USM was dried and weighed using a Mettler ME 30 microbalance ( $\pm 10 \mu \mathrm{g}$ on total residue). The saponifiable material was esterified by an excess of anhydrous $\mathrm{HCl}$-methanol solution, refluxing for $5 \mathrm{~min}$. After hydrolysis, fatty acid methyl esters (FAME) were recovered by extraction twice with $n$-hexane. After solvent-removing and drying, FAME were weighed and the fraction of FAME relative to total dry weight was calculated. FAME were then redissolved in $n$-hexane $\left(1 \mathrm{mg} \mathrm{ml}^{-1}\right)$ and an aliquot of $2 \mu \mathrm{l}$ was taken for gas chromatography analysis.

Gas chromatography of FAME. The gas chromatograph was a Girdel series 30 equipped with a flame ionization detector and using an injector-evaporator system. Two capillary glass columns coated with different stationary phases were used: (1) A polar phase:

Table 1. Phaeodactylum tricornutum and Dunaliella tertiolecta. Total fatty acid and unsaponifiable matter (USM) contents in cells cultured under phosphorus-sufficient (PS) and phosphorus-deficient (PD) batch conditions

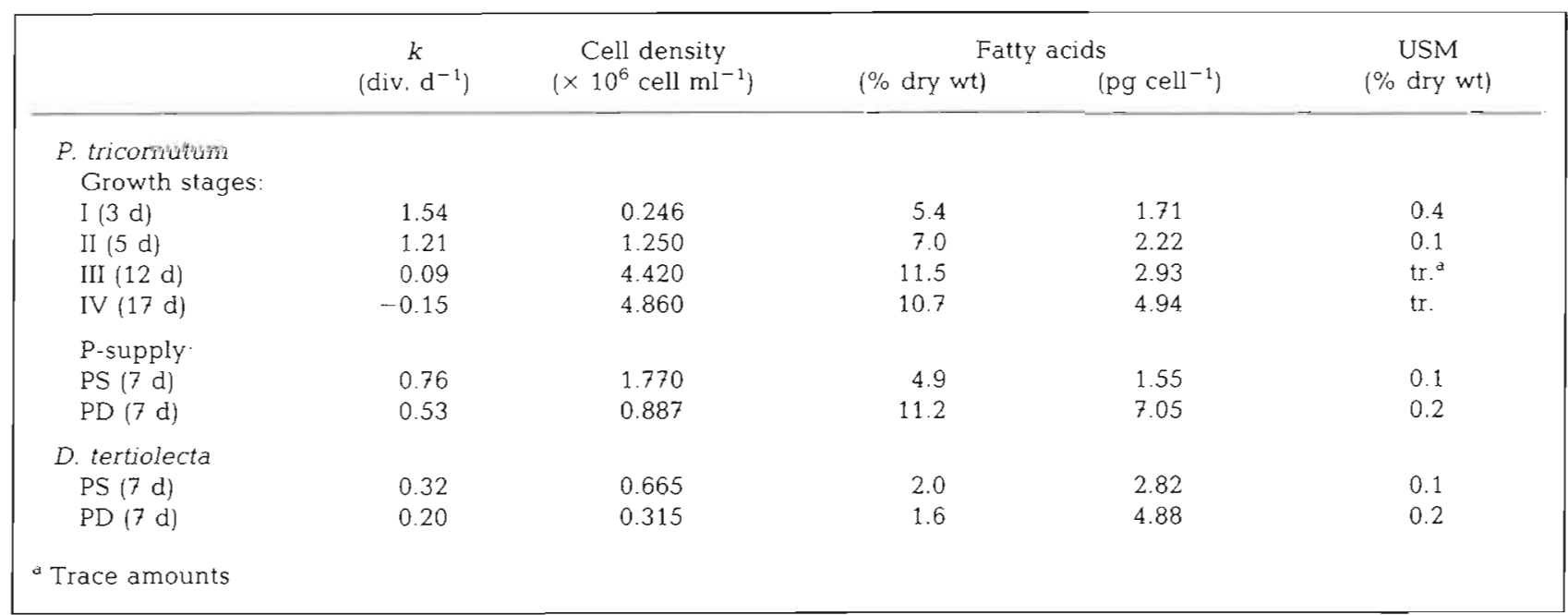


Carbowax $20 \mathrm{M}(\mathrm{L}=25 \mathrm{~m}$; i.d. $=0.3 \mathrm{~mm})$, used under the following conditions: oven temperature constant at $160{ }^{\circ} \mathrm{C}$; carrier gas: $\mathrm{He}, 0.5$ bar pressure. (2) A nonpolar phase: OV-1 $(\mathrm{L}=50 \mathrm{~m}$; i.d. $=0.3 \mathrm{~mm})$, used under the following conditions: oven temperature constant at $220^{\circ} \mathrm{C}$ during the first $20 \mathrm{~min}$ and then programmed from 220 to $250^{\circ} \mathrm{C}$ at $3 \mathrm{C}^{\circ} \mathrm{min}^{-1}$ until the end of analysis; carrier gas: He, 1.3 bar pressure. Temperatures of injector and detector were set at 295 and $280^{\circ} \mathrm{C}$ respectively.

The chromatograph was connected to a Shimadzu CR2AX recorder-integrator. FAME were determined by comparison of their retention times on both $\mathrm{OV}-1$ and Carbowax columns with those obtained from commercial vegetable and cod liver oils.

\section{RESULTS}

\section{Changes in lipid content with growth}

Growth-related changes in lipid content were studied in Phaeodactylum tricornutum culture and are reported in Table 1. Growth stages considered in this study correspond to the exponential growth phase (Stage I), the late-exponential phase (Stage II), the beginning (Stage III) and the very end (Stage IV) of the stationary phase.

As the culture grew, a significant quantitative increase in total fatty acids occurred, especially when the stationary phase was reached. By the end of the stationary phase (Stage IV), cells of Phaeodactylum tricornutum contained about 3 times as much fatty acids as during the exponential growth phase (Stage $I_{;}$ Table 1). Unsaponifiable material, which essentially contains sterols, hydrocarbons, terpenic alcohols and perhaps some pigments (Paoletti et al. 1976), decreased very rapidly as the culture grew older (Table 1).

In relative terms, the fatty acid composition of Phaeodactylum tricornutum during exponential growth phase (Stages I and II) was quite similar to that reported by Moreno et al. (1979). In particular, the fatty acid pattern was characterized by a large fraction of palmitic, palmitoleic and eicosapentaenoic acids (Table 2 ). Both palmitic and palmitoleic acids showed a substantial increase during growth and represented about three-quarters of total fatty acids by the end of the stationary phase. Conversely, other fatty acids rapidly decreased with culture age (e.g. stearic and behenic acids) whereas no significant change was observed in the relative proportion of myristic acid (Table 2). In the case of eicosapentaenoic acid and other polyunsatu-

Table 2. Phaeodactylum tricornutum. Fatty acid composition (\% of total) at different growth stages in enriched batch system and under phosphorus-sufficient (PS) and phosphorus-deficient (PD) condition

\begin{tabular}{|c|c|c|c|c|c|c|}
\hline \multirow{2}{*}{$\begin{array}{l}\text { Fatty acid } \\
\text { identity }\end{array}$} & \multicolumn{4}{|c|}{ Growth stages } & \multicolumn{2}{|c|}{ Phosphorus supply } \\
\hline & $\begin{array}{c}I \\
(3 d)\end{array}$ & $\begin{array}{c}\text { II } \\
(5 \mathrm{~d})\end{array}$ & $\begin{array}{c}\text { III } \\
(12 \mathrm{~d})\end{array}$ & $\begin{array}{c}\text { IV } \\
(17 \mathrm{~d})\end{array}$ & $\begin{array}{l}\text { PS } \\
(7 \mathrm{~d})\end{array}$ & $\begin{array}{l}\text { PD } \\
(7 \mathrm{~d})\end{array}$ \\
\hline C12:0 (lauric) & 0.4 & 1.0 & 1.1 & 1.8 & 1.1 & 0.4 \\
\hline C14:0 (myristic) & 5.8 & 5.3 & 5.7 & 6.3 & 6.8 & 5.6 \\
\hline C16:0 (palmitic) & 23.0 & 15.5 & 18.7 & 31.5 & 12.0 & 32.5 \\
\hline C16:1 (palmitoleic) & 24.2 & 24.1 & 32.7 & 43.0 & 22.4 & 40.4 \\
\hline $\mathrm{C} 16: 2$ & 5.8 & 11.7 & 12.2 & 4.5 & 9.2 & 1.1 \\
\hline $\mathrm{C} 16: 3$ & 1.0 & 1.8 & 0.9 & 0.4 & 5.3 & 0.4 \\
\hline C18:0 (stearic) & 3.8 & 0.4 & 1.3 & 0.4 & 0.4 & 0.8 \\
\hline C18:1 (oleic) & 1.4 & 0.6 & 0.7 & 2.1 & 0.5 & 0.6 \\
\hline C18:2 (linoleic) & 1.2 & 1.0 & 0.6 & 0.5 & 1.7 & 1.1 \\
\hline C18:3 (linolenic) & $\operatorname{tr}^{a}$ & 0.3 & 0.3 & tr. & 0.5 & 3.4 \\
\hline C18:4 & 0.7 & 0.4 & 0.4 & 1.3 & 3.9 & 0.5 \\
\hline $\mathrm{C} 18: \mathrm{n}^{\mathrm{b}}$ & 0.8 & 2.2 & 2.1 & tr. & 0.3 & 3.7 \\
\hline C20:5 (eicosapentaenoic) & 10.0 & 21.3 & 14.3 & 4.0 & 26.8 & 6.9 \\
\hline $\mathrm{C} 22: 0$ (behenic) & 0.7 & 0.1 & 0.3 & $\operatorname{tr}$ & 0.3 & 0.3 \\
\hline $\mathrm{C} 22: 6$ & 0.3 & 0.9 & 0.3 & $\operatorname{tr}$ & 1.1 & 0.3 \\
\hline Total fatty acids identified & 79.2 & 86.6 & 91.6 & 96.2 & 92.3 & 98.0 \\
\hline Unsaturation ratios: & & & & & & \\
\hline $\mathrm{C} 16: 1 / \mathrm{C} 16: 0$ & 1.05 & 1.55 & 1.75 & 1.36 & 1.86 & 1.24 \\
\hline C16 unsat./C16:0 & 1.35 & 2.42 & 2.45 & 1.52 & 3.07 & 1.29 \\
\hline C18 unsat./C18:0 & 1.10 & 11.25 & 3.15 & 10.25 & 17.25 & 11.62 \\
\hline Total unsat./total sat. & 1.35 & 2.88 & 2.38 & 1.40 & 3.48 & 1.47 \\
\hline
\end{tabular}


rated fatty acids (PUFA), it is interesting to note that they reached their maximal abundance during exponential growth phase. This significantly increased lipid unsaturation and a maximal degree of unsaturation was reached by the end of the exponential phase. Although C18 fatty acids represented a small proportion of the total, they also contributed to increase the unsaturation degree of total lipids, as suggested by the C18 unsaturated/C18:0 ratio (Table 2).

\section{Effect of phosphorus limitation on lipid content}

In this design of batch culture experiments, a significant influence of phosphorus-limitation can only be observed after $3 \mathrm{~d}$ growth in deficient medium (Siron unpubl.) because of phosphate internal pools. Phosphorus-limited cells were harvested after $7 \mathrm{~d}$ growth when cultures reached the end of the exponential phase. Quantitatively, the phosphorus-deficient (PD) culture of Phaeodactylum tricornutum contained, by the end of the exponential growth phase, more fatty acids than the phosphorus-sufficient (PS) culture. No similar trend was found for the chlorophyte Dunaliella tertiolecta when considering fatty acid proportion in dry weight (Table 1). However, a significant increase in the fatty acid content per cell was observed for both the diatom and the chlorophyte in PD conditions.

Chromatographic analysis clearly revealed that the PD diatom culture rapidly showed a fatty acid pattern

Table 3. Dunaliella tertiolecta. Fatty acid composition (\% of total) when cultured in enriched batch system after $7 \mathrm{~d}$ growth under phosphorus-sufficient (PS) and phosphorus-dificient (PD) conditions

\begin{tabular}{|crr|}
\hline \multirow{2}{*}{ Fatty acid identity } & \multicolumn{2}{c}{ Phosphorus supply } \\
& PS & PD \\
\hline C12:0 & 0.3 & 0.3 \\
C14:0 & 0.2 & 0.6 \\
C16:0 & 20.5 & 22.3 \\
C16:1 & 2.2 & 0.5 \\
C16:2 & 1.2 & 4.9 \\
C16:3 & 3.5 & 1.2 \\
C16:4 $18: 0$ & 9.2 & 12.4 \\
C18:1 & 0.8 & 1.0 \\
C18:2 & 0.4 & 12.1 \\
C18:3 & 7.4 & 6.6 \\
C18:4 & 38.5 & 27.5 \\
Total fatty acids identified & 8.8 & 4.0 \\
Unsaturation ratios: & 93.0 & 93.6 \\
C 16 unsat./C16:0 & & \\
C18:4+C18:3+C18:2/C18:1 & 136.75 & 3.15 \\
C18 unsat./C18:0 & 68.87 & 50.20 \\
Total unsat./total sat. & 3.26 & 2.86 \\
\hline
\end{tabular}

(Table 2) quite similar to that of a senescent culture. Indeed, proportions of fatty acids were comparable to those found by the end of the stationary phase in the enriched batch system (Stage IV, Table 2) although cell division continued at a slow rate $\left(k=0.53\right.$ div. $\left.d^{-1}\right)$. This altered pattern was characterized by the predominance of palmitoleic acid and, to a smaller extent, of palmitic acid which strongly contributed to decreasing the unsaturation of total lipids (Table 2). In the case of the chlorophyte, a difference in the fatty acid composition was also noted between cells grown in PS and PD media. This difference was accounted for principally by the relative increase of oleic acid to the detriment of linolenic acid which nevertheless remained the major fatty acid of Dunaliella tertiolecta (Table 3). Consequently, in relative unsaturation terms, the decline of polyunsaturated fatty acids was balanced by the relative accumulation of mono-unsaturated (oleic) acid. Finally, it is interesting to note that total lipid unsaturation of this microalga was only slightly affected by phosphorus limitation (compare unsaturation ratios in Table 3 ).

\section{DISCUSSION}

The increase with age in total fatty acids of Phaeodactylum tricornutum is in the same range as that reported for other diatoms (Collier 1970, Fisher \& Schwarzenbach 1978, Taguchi et al. 1987). It is probably related to lipid storage in cells when photosynthesis assimilation is carried out while cell division is blocked because of a nutritional deficiency. Under the growth conditions used in this batch culture experiment (initial $\mathrm{N} / \mathrm{P}$ ratio $=10$ in the enriched medium; Antia et al. 1975), nitrogen deficiency usually occurred by the end of growth. Small proportions of unsaponifiable lipids found in $P$. tricornutum are in agreement with data reported by Orcutt \& Patterson (1975) where sterols represented only $0.24 \%$ of the dry weight of this species. The high increase in C16 fatty acids in $P$. tricornutum when the batch culture grows older, also observed by Fisher \& Schwarzenbach (1978) for another diatom, Thalassiosira pseudonana, supports the idea that both palmitic and palmitoleic acids could actually represent an energy storage linked to extra production when the cell division is altered. Piorreck \& Pohl (1984), who carried out a similar study on 2 chlorophytes, did not note maximal abundance of PUFA during the exponential growth phase, but rather a constant decrease in most of PUFA during the growth course and consequently, a marked decrease of lipid unsaturation in old cells, as was found in the present study. Biosynthesis of PUFA takes place mainly during the phase of intense cellular activity and confirms their active part in the photosynthetic processes of $P$. tricor- 
nutum. Although $\mathrm{C} 18$ fatty acids are minor components in diatom lipids (Ackman et al. 1968, Collier 1970), they seem to play a key role in the lipid metabolism of $P$. tricornutum. Indeed, stearic and oleic acids could be the precursors of long chain PUFA since a decrease in their relative proportions was concurrent with the synthesis of PUFA during the exponential phase. Our results confirm the important role played by oleic acid as an intermediate component in the lipid metabolism of this diatom (Moreno et al. 1979). Therefore, in further lipid studies concerning this species, emphasis must be placed on these minor fatty acids because they are responsible for the increase in unsaturation degree of lipids during the exponential growth. They could also balance lipid unsaturation when lipid metabolism and subsequent fatty acid composition are altered by nutrient stress.

The accumulation of lipids by microalgae under nutritional stress has often been reported (Opute 1974, Shifrin \& Chisholm 1981, Tornabene et al. 1983, Suen et al. 1987). However, with particular attention to chlorophytes, it must be noted that Ben-Amotz et al. (1985) found no lipid accumulation in nitrogendeficient cultures of Dunaliella salina, consistent with our results. According to Parrish \& Wangersky (1987) the only available data concerning the lipid response of Phaeodactylum tricornutum to a nutritional (nitrogen) stress - the lipid metabolism of this species seems sensitive to the growth conditions (batch or continuous systems). Thus, nutrient deficiency might not be the only factor influencing lipid storage. Inasmuch as light can also influence the fatty acid composition of microalgae (Orcutt \& Patterson 1974), it is possible that changes in lipid composition related to the age of cultures could be partly attributed to a significant decline in light intensity when high cell densities are reached in batch cultures. Although the different lipid classes were not studied here, the storage of palmitic and palmitoleic acids when the diatom is cultured under PD conditions can be related to the accumulation of triglycerids to the detriment of phospholipids (Parrish \& Wangersky 1987). Indeed, under nutrient limitation, since nitrogen - as well as phosphorus - participates in the elaboration of phospholipids, lipid synthesis would be oriented towards the storage of relatively saturated triglycerids and neutral lipids (Tornabene et al. 1983, Piorreck et al. 1984, Suen et al. 1987), as was observed in this study. Phosphorus limitation leads to a significant decrease in PUFA altering the global unsaturation of microalgal lipids. In particular, biosynthesis of eicosapentaenoic acid is rapidly reduced by phosphorus deficiency. This essential fatty acid is one of the most important in marine lipids and its relative proportion varies about from 5 to $20 \%$ in commercial fish oils (Ackman 1982).
Changes in the fatty acid composition of Dunaliella tertiolecta do not seem to be directly linked to a premature ageing of the PD culture. Indeed, in a similar study conducted on this species, Ackman et al. (1968) observed a reversed trend in the fatty acid pattern in aged cultures. The accumulation of oleic acid parallel to the reduction of cell division observed in PD medium seems to confirm the key function played by this fatty acid (Piorreck et al. 1984, Ben-Amotz et al. 1985) in the microalgal processes of cell division. Since the desaturation of oleic acid requires oxygen and essentially takes place inside chloroplasts, accumulation observed here might be the consequence of an alteration of photosynthetic assimilation processes in D. tertiolecta. The fact that total lipid unsaturation is very slightly affected, despite an alteration of fatty acid distribution, gives evidence that this microalga is able to maintain an unsaturation level of lipids during nutritional deficiency. The quality of lipids accumulated under phosphorus limitation is however altered with regard to the lower proportion of the essential linolenic acid and other PUFA. No similar unsaturation balance was observed for the diatom revealing a drastic deterioration of the lipid quality. Such differences in the lipid response could have direct ecological implications in the alteration of the specific composition of microalgal communities submitted to local phosphorus limitation, and thus also in higher trophic levels with regard to the available phytoplanktonic pool of essential fatty acids.

Particular attention is required when comparing lipid results from microalgae grown in batch cultures, for while culture conditions are generally well defined and controlled, culture age is not always specified and this could have a considerable influence on the lipid composition of microalgae as clearly demonstrated in this study. Finally, it is important to point out that the lipid accumulation of some microalgae cultured under nutrient stresses could be used as a means of obtaining vegetable oil for industrial applications (Shifrin \& Chisholm 1980). For both the diatom and the chlorophyte that were considered here, results display decreases in lipid unsaturation and in the mean of fatty acid carbon chains simultaneously with increases in the fatty acid content. Consequently, when considering such bioenergetic prospects, perfect control of the potential energy contained in vegetable oils thus formed should not be expected.

Acknowledgements. This study was supported by a grant (DGRST No, 82-217) from the French Ministry of Science and Technology to R. Siron. Drs Ucciani and Mallet of the StJérôme University (Marseille) are gratefully acknowledged for their valuable advice concerning lipid analysis. R.S. thanks Drs J.-F. Rontani and R. Morales-Loo for their efficient help in the laboratory and Dr S. Roy for critically reviewing this manuscript. 


\section{LITERATURE CITED}

Ackman, R. G. (1967). Characteristics of the fatty acid composition and biochemistry of some fresh-water fish oils and lipids. Comp. Biochem. 22: 907-922

Ackman, R. G. (1982). Fatty acid composition of fish oils. In: Barlow, S. M., Stansby, M. E. (eds.) Nutritional evaluation of long-chain fatty acids in fish oil. Academic Press, New York, p. 25-88

Ackman, R. G., Tocher, C. S., McLachlan, J. (1968). Marine phytoplankter fatty acids. J. Fish. Res. Bd Can. 25: $1603-1620$

Antia, N. J., Berland, B. R., Bonin, D. J., Maestrini, S. Y, (1975). Comparative evaluation of certain organic and inorganic sources of nitrogen for phototrophic growth of marine microalgae. J. mar. biol. Ass. U.K. 55: 519-539

Ben-Amotz, A., Tornabene, T. G., Thomas, W. H. (1985). Chemical profile of selected species of microalgae with emphasis on lipids. J. Phycol. 21: 72-81

Berland, B. R. (1966). Contribution à l'étude des cultures de diatomées marines. Recl. Trav. Stn mar. Endoume 40: 1-82

Berland, B. R., Bonin, D. J., Maestrini, S. Y (1980). Azote ou phosphore: considérations sur le paradoxe nutritionnel de la mer Méditerranée. Oceanologica Acta 3: 135-142

Collier, A. (1970). Fatty acids in certain plankton organisms. In: Lauff, G. H. (ed.) Estuaries: nutrients and biological production. American Association for the Advancement of the Science, Washington D.C., p. 353-360

Fisher, N. S., Schwarzenbach, R. P. (1978). Fatty acid dynamics in Thalassiosira pseudonana (bacillariophyceae): implications for physiological ecology. J. Phycol. 14: 143-150

Kaellqvist, T. (1988). Nitrogen or phosphorus - what is the limiting nutrient in coastal areas. Examples from Norwegian fjords. Vatten $44: 11-18$

Kanasawa, A., Teshima, S., Ono, K., Chalayondeja, K. (1979). Biosynthesis of fatty acids from acetate in the prawns, Peneaeus monodon and Peneaeus merguensis. Mem. Fac. Fish. Kagoshima Univ. 28: 21-28

Lee, R. F., Nevenzel, J. C., Paffenhoffer, G. A. (1971). Importance of wax-esters and other lipids in the marine food chain: phytoplankton and copepods. Mar. Biol. 9: 99-108

Luddy, F. E., Barford, R. A., Riemenschneider, R. W. (1960). Direct conversion of lipid components to their fatty acid methyl esters. J. Am. Oil Chem. Soc. 37: 447-451

Mayzaud, P., Eaton, C. A., Ackman, R. G. (1976). The occurrence and distribution of octadecapentaenoic acid in a natural plankton population. A possible food chain index. Lipids 12: 858-862

Moreno, V J., De Moreno, J. E. A., Brenner, R. R. (1979). Biosynthesis of unsaturated fatty acids in the diatom: Phaeodactylum tricornutum. Lipids 14: 15-19

Myers, $V$ B., Iverson, R. I. (1981). Phosphorus and nitrogen limited phytoplankton productivity in northeastern Gulf of Mexico coastal estuaries. In: Nielson, B. J., Cronin, L. E. (eds.) Estuaries and nutrients. Humana Press, Clifton, N. J., p. 569-582

Opute, F. I. (1974). Studies on fat accumulation in Nitzschia palea Kütz. Ann. Bot. 38: 889-902

Orcutt, D. M., Patterson, G. W (1974). Effect of light intensity upon Nitzschia closternium (Cylindrotheca fusiformis). Lipids 9: 1000-1003

This article was submitted to the editor
Orcutt, D. M., Patterson, G. W. (1975). Sterol, fatty acid and elemental composition of diatoms grown in chemically defined media. Comp. Biochem. Physiol. 50: 579-583

Paoletti, C., Pushparaj, B., Florenzano, G., Cepello, P., Lercker, G. (1976). Unsaponifiable matter of green and blue-green algal lipids as a factor of biochemical differenciation of their biomasses. I: Total unsaponifiable and hydrocarbon fraction. Lipids 11: 258-265

Parrish, C. G., Wangersky, P. J. (1987). Particulate and dissolved lipid classes in cultures of Phaeodactylum tricomutum grown in cage culture turbidostats with a range of nitrogen supply rates. Mar. Ecol. Prog. Ser. 35: 119-128

Piorreck, M., Baasch, K. H., Pohl, P. (1984). Biomass production, total protein, chlorophylls, lipids and fatty acids of freshwater green and blue-green algae under different nitrogen regimes. Phytochemistry 23: 207-216

Piorreck, M., Pohl, P. (1984). Formation of biomass, total protein, chlorophylls, lipids and fatty acid in green and bluegreen algae during one growth phase. Phytochemistry 23 : $217-223$

Pohl, P., Zurheide, F. (1979). Fatty acids and lipids in marine algae and the control of their biosynthesis by invironmental factors. In: Hope, H. A., Levring, T., Tanaka, Y (eds.) Marine algae in pharmaceutical science. W. de Gruyter, Berlin, p. 433-523

Saliot, A., Barbier, M. (1973). Acides gras, hydrocarbures et stérols dissous dans l'eau de mer. In: Tissot, B., Bienner, F. (eds.) Advances in organic geochemistry, 6ème congrès international de géochimie organique. Technip, RueilMalmaison, p. 607-617

Schneider, H., Gelpi, E., Benett, E. O., Oro, J. (1970). Fatty acids of geochemical significance in microscopic algae. Phytochemistry: 613-617

Shifrin, N. S., Chisholm, S. W (1980). Phytoplankton lipids: environmental influences on production and possible commercial applications. In: Shelef, G., Soeder, C. J. (eds.) Algae biomass: production and use. Elsevier North-Holland Biomedical Press, Amsterdam, p. 627-645

Shifrin, N. S., Chisholm, S. W. (1981). Phytoplankton lipids: interspecific differences and effects of nitrate, silicate and light-dark cycles. J. Phycol. 17: 374-384.

Siron, R., Giusti, G. (1985). Fatty acid composition of plankton from the Gulf of Fos-sur-mer. C. r. hebd. Séanc. Acad. Sci., Paris, série III 301: 191-194

Siron, R., Morales-Loo, M. del R., Berland, B. (1986). Effects of soluble petroleum compounds on the growth of two planktonic algae. C. r. hebd. Séanc. Acad. Sci., Paris, série III 303: 349-352

Suen, Y., Hubbard, J. S., Holzer, G., Tornabene, T. G. (1987). Total lipid production of the green alga Nannochloropsis Sp. QII under different nitrogen regimes. J. Phycol. 23: 289-296

Taguchi, S., Hirata, J. A., Laws, E. A. (1987). Silicate deficiency and lipid synthesis of marine diatoms. J. Phycol. 23: 260-267

Tornabene, T G., Holzer, G., Lien, S., Burris, N. (1983). Lipld composition of the nitrogen starved green alga Neochloris oleoabundans. Enzyme Microb. Technol. 5: 435-440

Wood, B. J. B. (1874). Fatty acids and saponifiable matenal. In: Stewart, W. D. P. (ed.) Algal physiology and biochemistry, Chap. 8. Blackwell, Oxford, p. 236-265

Manuscript first received: November 15, 1988

Revised version accepted: April 19, 1989 